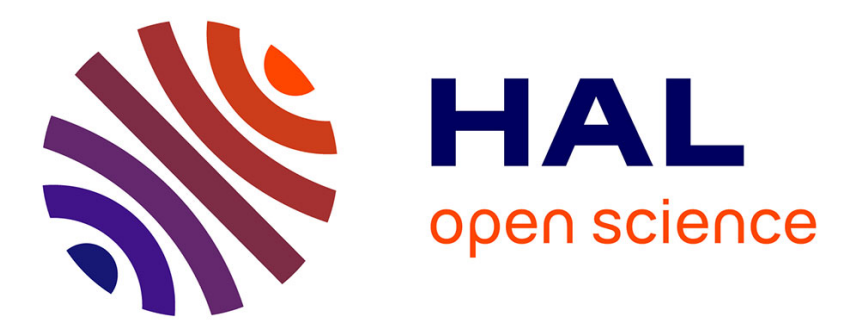

\title{
Conformational Effect on the Large Amplitude Motions of 3,4-Dimethylanisole Explored by Microwave Spectroscopy
}

\author{
Lynn Ferres, Jenny Cheung, Wolfgang Stahl, Ha Vinh Lam Nguyen
}

\section{To cite this version:}

Lynn Ferres, Jenny Cheung, Wolfgang Stahl, Ha Vinh Lam Nguyen. Conformational Effect on the Large Amplitude Motions of 3,4-Dimethylanisole Explored by Microwave Spectroscopy. Journal of Physical Chemistry A, 2019, 123 (16), pp.3497-3503. 10.1021/acs.jpca.9b00029 . hal-03183062

\section{HAL Id: hal-03183062 https://hal.science/hal-03183062}

Submitted on 26 Mar 2021

HAL is a multi-disciplinary open access archive for the deposit and dissemination of scientific research documents, whether they are published or not. The documents may come from teaching and research institutions in France or abroad, or from public or private research centers.
L'archive ouverte pluridisciplinaire HAL, est destinée au dépôt et à la diffusion de documents scientifiques de niveau recherche, publiés ou non, émanant des établissements d'enseignement et de recherche français ou étrangers, des laboratoires publics ou privés. 


\title{
Conformational Effect on the Large Amplitude
}

\section{Motions of 3,4-Dimethylanisole Explored by}

\section{Microwave Spectroscopy}

\author{
Lynn Ferres, ${ }^{a}$ Jenny Cheung, ${ }^{a}$ Wolfgang Stahl, ${ }^{a}$ Ha Vinh Lam Nguyen ${ }^{{ }^{*}}$
}

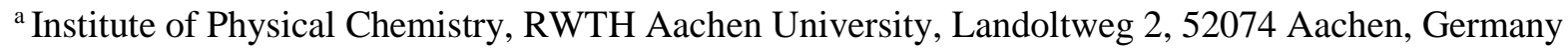

${ }^{\mathrm{b}}$ Laboratoire Interuniversitaire des Systèmes Atmosphériques (LISA), CNRS UMR 7583, Université

Paris-Est Créteil, Université Paris Diderot, Institut Pierre Simon Laplace, 61 avenue du Général de Gaulle, 94010 Créteil Cedex, France
\end{abstract}

*Corresponding author: lam.nguyen@lisa.u-pec.fr 


\section{ABSTRACT}

The microwave spectrum of 3,4-dimethylanisole, a molecule containing three methyl groups allowing for internal rotation, was recorded using a pulsed molecular jet Fourier transform microwave spectrometer operating in the frequency range from 2.0 to $26.5 \mathrm{GHz}$. Quantum chemical calculations yielded two conformers with a trans and a cis configuration of the methoxy group, both of which were assigned in the experimental spectrum. Torsional splittings due to the internal rotations of two methyl groups attached to the aromatic ring were resolved and analysed. The rotation-torsional transitions could be reproduced to measurement accuracy, yielding well-determined rotational and internal rotation parameters. The torsional barriers of the methyl groups at the meta and para position were deduced to be 430.00(37) and 467.90(17) $\mathrm{cm}^{-1}$, respectively, for the cis-conformer. The respective values for the trans-conformer are 499.64(26) and 533.54(22) $\mathrm{cm}^{-1}$. A labelling scheme for the $\mathrm{G}_{18}$ group written as the semidirect product $\left(C_{3}^{I} \otimes C_{3}^{I}\right) \otimes C_{s}$ was introduced.

\section{INTRODUCTION}

High resolution studies on small organic molecules undergoing large amplitude motions (LAMs) have received growing interest in the last decades, because the intramolecular dynamics are not only interesting but also fundamentally important investigations in physics and chemistry. The combination of microwave spectroscopy and quantum chemical calculations is an excellent tool to determine the structure and LAM parameters of a molecule, as demonstrated in many cases previously published in the literature. ${ }^{1-4}$ The two main types of LAMs are inversion tunneling and internal rotation. Some recent investigations on inversion tunneling are, e.g., the proton tunneling in diethyl amine ${ }^{5}$, the ring tunneling in phenyl formate ${ }^{6}$ and acetanilide, ${ }^{7}$ or the intra-molecular hydrogen transfer in 5 -methyltropolone. ${ }^{8}$ There are a 
vast amount of examples for internal rotation, such as the methyl internal rotation in 2,4-difluorotoluene, ${ }^{9}$ methyl mercaptane, ${ }^{10}$ and 5-methyl furfuran. ${ }^{11}$

Coupled LAMs are reported much more rarely. Methyl amine is one of the classical cases where the interplay between the $\mathrm{NH}_{2}$ tunneling and the methyl internal rotation remains a challenge until today. ${ }^{12}$ Investigations on several molecules undergoing internal rotations of two methyl tops are available, e.g. refs. 13-17, however, in only few cases such as the three isomers of dimethylbenzaldehyde (DMBA), ${ }^{18} o$ - and $m$-xylene, ${ }^{19,20}$ and 2,3-dimethylanisole ${ }^{21}$ the methyl rotors are attached to a phenyl ring. If they are located in the neighboring ring positions, steric effect might strengthen the coupling between the LAMs and complicate the spectrum. ${ }^{18,21}$

The three isomers of mono-methylanisole have been thoroughly studied by microwave spectroscopy, yielding highly accurate values for the barriers to internal rotation. For $o$ - methylanisole, the cis and the trans conformer of $m$-methylanisole, and $p$-methylanisole, the respective barrier heights are $444.05(41) \mathrm{cm}^{-1},{ }^{22} 55.7693(90) \mathrm{cm}^{-1}$ and $36.6342(84) \mathrm{cm}^{-1},{ }^{23}$ as well as $48.7400(93) \mathrm{cm}^{-1} \cdot{ }^{24}$ The potential barriers of the methyl groups in dimethylanisoles cannot be directly derived from those of the mono-methylanisoles. Currently, studies on only one example of dimethylanisole are available in the literature, and many fitting problems occur because of the strong LAM coupling ${ }^{21}$. Therefore, we decide to study a related case, 3,4-dimethylanisole (34DMA), where the two methyl tops are also in neighboring positions.

Like the other members in the dimethylanisole family, 34DMA features a methoxy group and two methyl groups attached to the aromatic ring. Therefore, 34DMA possesses four degrees of freedom of interest: the orientation of the methoxy group and the internal rotations of three methyl groups. Each of the latter results in three partially energetically degenerated states, and the former yields two conformers of different energies. While the internal rotation of the methoxy methyl group is rather isolated, the other three degrees of freedom are connected 
by the conjugated $\pi$-system in the ring. Therefore, 34DMA is ideally suited to study the conformational effect (i.e. the methoxy orientation) on the internal rotations of the methyl groups in combination with the coupling between these LAMs.

\section{THEORY}

Quantum Chemical Calculations. The rotations about the $\mathrm{C}_{3}-\mathrm{C}_{19}, \mathrm{C}_{4}-\mathrm{C}_{10}$, and $\mathrm{O}_{14}-\mathrm{C}_{15}$ bonds (for atom numbering see Figure 1) correspond to the internal rotation of three methyl groups and do not create new conformations. Therefore, the conformational landscape of 34DMA is determined by the orientation of the methoxy group. By rotating this group about the $\mathrm{C}_{1}-\mathrm{O}_{14}$ bond, a potential energy curve was calculated at the B3LYP/6-311++G(d,p) level of theory using the Gaussian09 program package, ${ }^{25}$ where the dihedral angle $\alpha=\angle\left(\mathrm{C}_{2}, \mathrm{C}_{1}, \mathrm{O}_{14}, \mathrm{C}_{15}\right)$ was varied in steps of $10^{\circ}$, while all other degrees of freedom were allowed to relax during the optimization process. We chose this level because it has yielded reliable results in the studies of all three isomers of mono-methylanisoles. ${ }^{22-24}$ The potential energy curve was parameterized with the Fourier coefficients given in Table S1 in the Supporting Information (SI), then drawn as a contour plot depicted in Figure 2. 

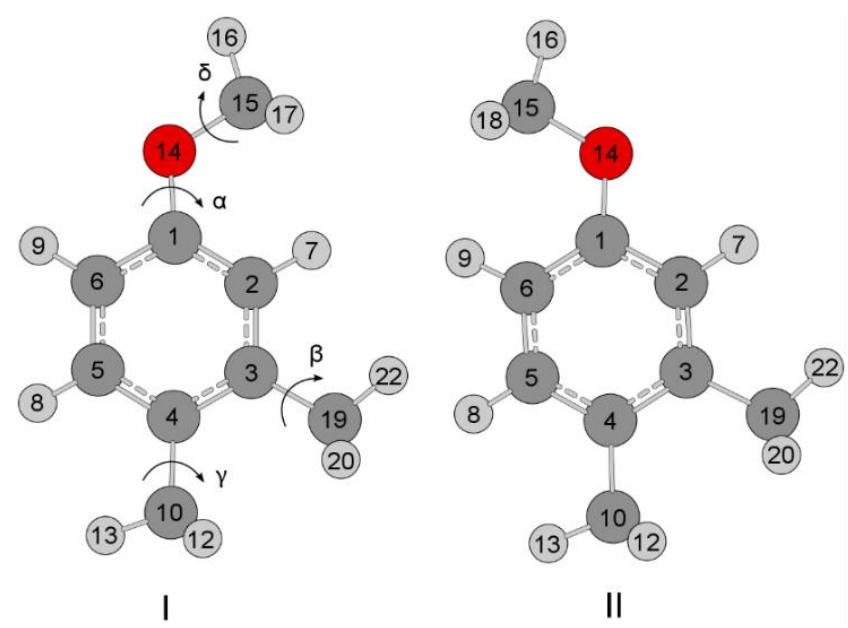

Figure 1. Molecular structures of the two conformers cis (I) and trans (II) of 34DMA optimized at the B3LYP/6-311++G(d,p) level of theory. The trans conformer with an absolute energy of $E_{Z P E}=-425.334355$ Hartree including zero-point energy corrections is $0.91 \mathrm{~kJ} \cdot \mathrm{mol}^{-1}$ lower in energy than the cis conformer $\left(\mathrm{E}_{\mathrm{ZPE}}=-425.334008\right.$ Hartree $)$. The dihedral angles are defined as $\alpha=$ $\angle\left(\mathrm{C}_{2}, \mathrm{C}_{1}, \mathrm{O}_{14}, \mathrm{C}_{15}\right), \beta=\angle\left(\mathrm{C}_{2}, \mathrm{C}_{3}, \mathrm{C}_{19}, \mathrm{H}_{22}\right), \gamma=\angle\left(\mathrm{C}_{5}, \mathrm{C}_{4}, \mathrm{C}_{10}, \mathrm{H}_{13}\right)$, and $\delta=\angle\left(\mathrm{H}_{16}, \mathrm{C}_{15}, \mathrm{O}_{14}, \mathrm{C}_{1}\right)$, corresponding to rotations about the $\mathrm{C}_{1}-\mathrm{O}_{14}, \mathrm{C}_{3}-\mathrm{C}_{19}, \mathrm{C}_{4}-\mathrm{C}_{10}$, and $\mathrm{O}_{14}-\mathrm{C}_{15}$ bonds, respectively.

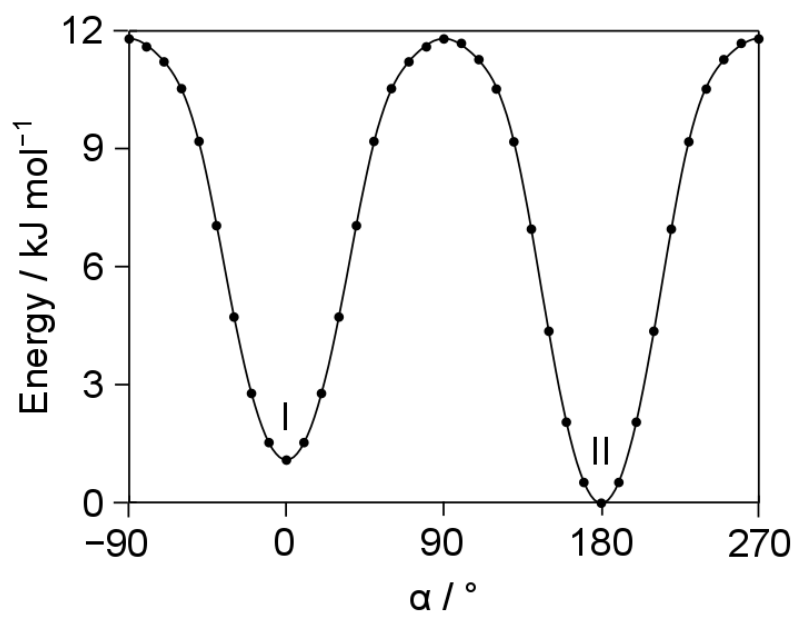

Figure 2. The potential energy curve of 34DMA obtained by rotating the methoxy group about the $\mathrm{O}_{14}-\mathrm{C}_{15}$ bond by varying the dihedral angle $\alpha=\angle\left(\mathrm{C}_{2}, \mathrm{C}_{1}, \mathrm{O}_{14}, \mathrm{C}_{15}\right)$ in steps of $10^{\circ}$ and optimized at the B3LYP/6-311++G(d,p) level of theory. The relative energies with respect to the lowest energy conformation with $E_{\min }=-425.5215701$ Hartree are given. Two stable $\mathrm{C}_{\mathrm{s}}$ conformers $\mathbf{I}\left(\right.$ cis, $E_{\text {rel }}=1.09$ $\mathrm{kJ} \cdot \mathrm{mol}^{-1}$ ) and II (trans, $\left.E_{\text {rel }}=0 \mathrm{~kJ} / \mathrm{mol}\right)$ were found at $\alpha=0^{\circ}$ and $180^{\circ}$, respectively. 
Two minima with an energy difference of $1.09 \mathrm{~kJ} \cdot \mathrm{mol}^{-1}$ exist at $\alpha=0^{\circ}$ and $180^{\circ}$, which will be referred to as cis-34DMA (conformer I) and trans-34DMA (conformer II), respectively, henceforward. The geometries of these minima were used as starting points for fulloptimizations also at the B3LYP/6-311++G(d,p) level of theory. The optimized structures are illustrated in Figure 1. The Cartesian coordinates of both conformers are available in Table S2 in the SI. Harmonic frequency calculations were carried out subsequently, which confirmed that both minima are stable conformers and not saddle points. For trans-34DMA the calculated dipole moment components are $\mu_{a}=0.18 \mathrm{D}, \mu_{b}=-0.90 \mathrm{D}$, and $\mu_{c}=0.00 \mathrm{D}$. The respective values for the cis conformer are $\mu_{a}=-0.15 \mathrm{D}, \mu_{b}=1.58 \mathrm{D}$, and $\mu_{c}=0.00 \mathrm{D}$. Therefore, strong $b$-type, weak $a$-type, and no $c$-type transitions are expected in the microwave spectrum. The optimized dihedral angles are $\alpha=0.15^{\circ}, \beta=0.02^{\circ}, \gamma=0.04^{\circ}, \delta=179.88^{\circ}$ for cis-34DMA, and $\alpha=179.97^{\circ}, \beta=0.01^{\circ}, \gamma=0.01^{\circ}, \delta=-179.96^{\circ}$ for trans-34DMA (II). Because the predicted energy difference obtained from structure optimizations is only $0.91 \mathrm{~kJ} \cdot \mathrm{mol}^{-1}$, both conformers might be present in the experimental spectrum under supersonic jet conditions. Anharmonic frequency calculations were carried out to provide centrifugal distortion constants and rotational constants for the vibrational ground state.

To calculate the barriers to internal rotation of the ring methyl groups at the meta and para positions, the dihedral angles $\beta=\angle\left(\mathrm{C}_{2}, \mathrm{C}_{3}, \mathrm{C}_{19}, \mathrm{H}_{22}\right)$ and $\gamma=\angle\left(\mathrm{C}_{5}, \mathrm{C}_{4}, \mathrm{C}_{10}, \mathrm{H}_{13}\right)$, respectively, were each varied in a grid of $10^{\circ}$, while all other coordinates were optimized at the B3LYP/6--311++G(d,p) level. Threefold torsional potentials were obtained, as illustrated in Figure 3. The Fourier coefficients of these potential energy curves are available in Table S3 in the SI. For the meta methyl group, the $V_{3}$ potential is $364.26 \mathrm{~cm}^{-1}$ for the cis conformer and $421.38 \mathrm{~cm}^{-1}$ for the trans conformer. The respective values for the para methyl group are slightly higher $\left(393.22 \mathrm{~cm}^{-1}\right.$ and $\left.444.70 \mathrm{~cm}^{-1}\right)$. Geometry optimizations to a first-order transition state of the methyl groups using the Berny algorithm ${ }^{26}$ yielded essentially similar 
values $\left(366.90 \mathrm{~cm}^{-1}\right.$ and $420.56 \mathrm{~cm}^{-1}$ for the meta methyl group as well as $394.66 \mathrm{~cm}^{-1}$ and $447.09 \mathrm{~cm}^{-1}$ for the para methyl group).
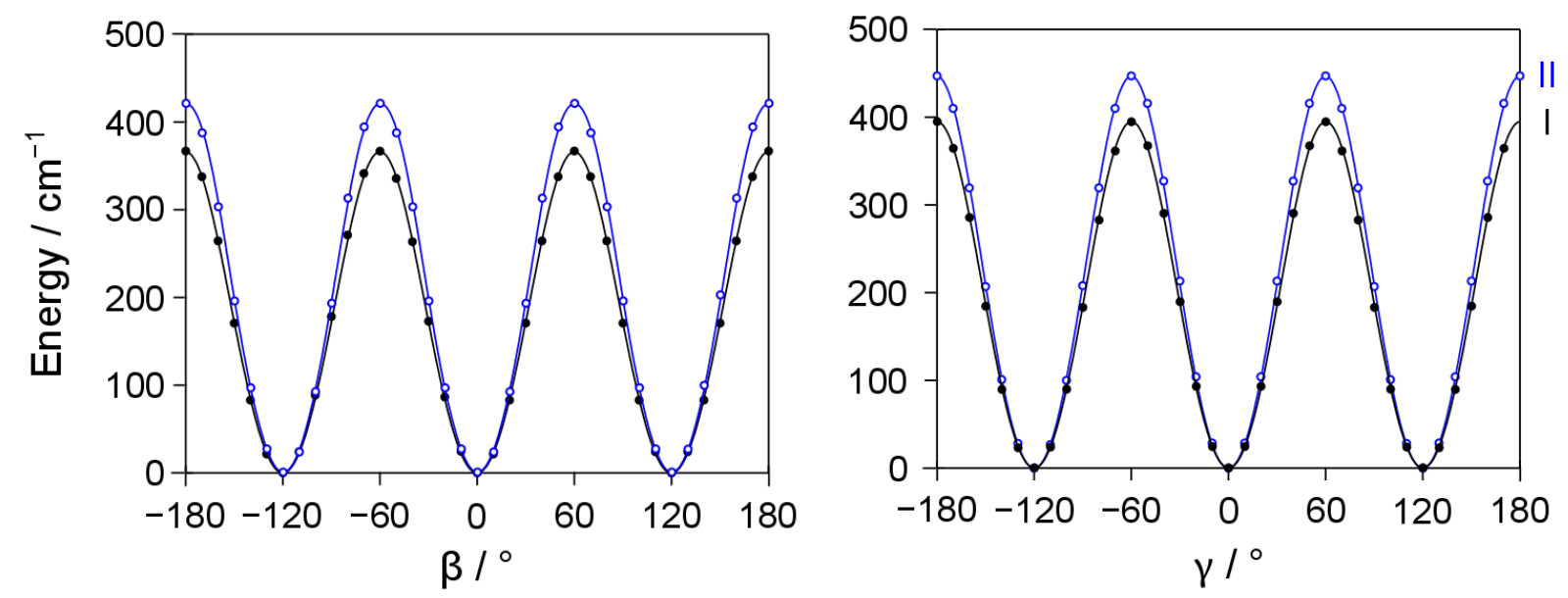

Figure 3. Threefold torsional potentials obtained by varying the dihedral angles $\beta=\angle\left(\mathrm{C}_{2}, \mathrm{C}_{3}, \mathrm{C}_{19}, \mathrm{H}_{22}\right)$ and $\gamma=\angle\left(\mathrm{C}_{5}, \mathrm{C}_{4}, \mathrm{C}_{10}, \mathrm{H}_{13}\right)$, corresponding to the internal rotations of the meta and para methyl groups, respectively. Left hand side: For the meta methyl group, the barrier height is $364.26 \mathrm{~cm}^{-1}$ for the cis conformer (I) and $421.38 \mathrm{~cm}^{-1}$ for the trans conformer (II). Energies relative to the lowest conformations with their absolute energies of $E=-425.5211543$ Hartree (cis-34DMA) and $E=-425.5215700$ Hartree (trans-34DMA) are given. Right hand side: For the para methyl group, the respective barrier heights are $393.22 \mathrm{~cm}^{-1}$ and $444.70 \mathrm{~cm}^{-1}$; the respective absolute energies are -425.5211543 Hartree and -425.5215701 Hartree.

To study the coupling between the internal rotations of the two methyl groups, a two-dimensional potential energy surface (2D-PES) of the cis conformer depending on $\beta$ and $\gamma$ was calculated by varying these dihedral angles in a $10^{\circ}$ grid. The potential energies were parameterized with a 2D Fourier expansion based on terms representing the correct symmetry of $\beta$ and $\gamma$. The Fourier coefficients are summarized in Table S4 in the SI. The PES drawn as a color contour plot given in Figure S1 using these coefficients shows almost no potential 
coupling between $\beta$ and $\gamma$. Due to the limited computational resources, the PES calculations were not redone for the trans conformer.

For the methoxy methyl group, the barrier to internal rotation was reported to be about $1200 \mathrm{~cm}^{-1}$ in the case of anisole. ${ }^{27}$ Therefore, a barrier of over $1000 \mathrm{~cm}^{-1}$ is expected for both conformers of 34DMA. Previous investigations on the three isomers of monomethylanisole $\mathrm{e}^{22-24}$ as well as on 2,3-dimethylanisole ${ }^{21}$ have proven that torsional splittings arising from the methoxy methyl group are not observable with our experimental resolution and can be neglected.

\section{Group theory.}

Symmetry labels. Considering the configuration of 34DMA which has a $\mathrm{C}_{\mathrm{s}}$ symmetry and two inequivalent methyl rotors, it is well-known that the molecular symmetry group of such a molecule is $\mathrm{G}_{18}$ with the character table given in Table $1 .^{28,29}$

Different symmetry labels are currently in use which arise from different product decompositions of $\mathrm{G}_{18}$. One of them is based on the direct product $C_{3 v}^{(1)} \otimes C_{3 v}^{(2)}$, and the symmetry species of $\mathrm{G}_{18}$ are labeled with the labels of both subgroups $C_{3 v}^{(1)}$ and $C_{3 v}^{(2)}$ as given in column S3 of Table $1 .{ }^{28}$ Using the permutation-inversion group formalism, Bunker and Jensen derived a character table of methylsilane, where the different species are denoted by their degeneracy letter A, E, and a running index ${ }^{29}$ as shown in column S2 of Table 1. 
Table 1. Character table of the molecular symmetry group $\mathrm{G}_{18}$ of 34DMA.

\begin{tabular}{llllcccccc}
\hline \multicolumn{2}{c}{$\mathrm{G}_{18}{ }^{\mathrm{a}}$} & & $\mathrm{E}$ & $\beta$ & $\alpha$ & $\alpha \beta$ & $\alpha \beta^{2}$ & $\sigma$ & \\
\multicolumn{2}{c}{ Equiv. rot. } & & $\mathrm{R}_{0}$ & $\mathrm{R}_{0}$ & $\mathrm{R}_{0}$ & $\mathrm{R}_{0}$ & $\mathrm{R}_{0}$ & $\mathrm{R}_{2 \mathrm{c}}$ & \\
\hline $\mathrm{S}^{\mathrm{c}}$ & $\mathrm{S}^{\mathrm{d}}$ & $\mathrm{S}^{\mathrm{e}}$ & $1^{\mathrm{f}}$ & $2^{\mathrm{f}}$ & $2^{\mathrm{f}}$ & $2^{\mathrm{f}}$ & $2^{\mathrm{f}}$ & $9^{\mathrm{f}}$ & $\mathrm{wt}^{\mathrm{g}}$ \\
$(00) \cdot \mathrm{A}^{\prime}$ & $\mathrm{A}_{1}$ & $\mathrm{~A}$ & 1 & 1 & 1 & 1 & 1 & 1 & 1024 \\
$(00) \cdot \mathrm{A}^{\prime \prime}$ & $\mathrm{A}_{2}$ & $\mathrm{~B}$ & 1 & 1 & 1 & 1 & 1 & -1 & 1024 \\
$(10) \cdot \mathrm{A}$ & $\mathrm{E}_{1}$ & $\mathrm{E}_{\alpha}$ & 2 & 2 & -1 & -1 & -1 & 0 & 1024 \\
$(01) \cdot \mathrm{A}$ & $\mathrm{E}_{2}$ & $\mathrm{E}_{\beta}$ & 2 & -1 & 2 & -1 & -1 & 0 & 1024 \\
$(11) \cdot \mathrm{A}$ & $\mathrm{E}_{3}$ & $\mathrm{E}_{\gamma}$ & 2 & -1 & -1 & 2 & -1 & 0 & 512 \\
$(12) \cdot \mathrm{A}$ & $\mathrm{E}_{4}$ & $\mathrm{E}_{\delta}$ & 2 & -1 & -1 & -1 & 2 & 0 & 512 \\
\hline \hline
\end{tabular}

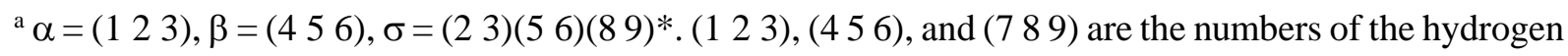
atoms of the meta-, para-, and methoxy methyl groups, respectively, where the hydrogen 1, 4, and 7 are located on the symmetry plane. Mapping to the atom numbering in Figure $1,1 \equiv 22,2 \equiv 21,3 \equiv 20$, $4 \equiv 13,5 \equiv 11,6 \equiv 12,7 \equiv 16,8 \equiv 18,9 \equiv 17 .{ }^{\mathrm{b}}$ Equivalent rotations of the rotation group. ${ }^{\mathrm{c}}$ Symmetry labels based on the semi-direct product $\left(C_{3}^{I} \otimes C_{3}^{I}\right) \otimes C_{S}$, see ref. 30. ${ }^{\mathrm{d}}$ Symmetry labels according to ref. [29]. ${ }^{\mathrm{e}}$ Symmetry labels based on the direct product $C_{3 v}^{(1)} \otimes C_{3 v}^{(2)}$, see ref. $28 .{ }^{\mathrm{f}}$ Number of elements in class. ${ }^{\mathrm{g}}$ Spin statistical weight.

We prefer a more instructive third labeling scheme (column S1) for 34DMA, for which $\mathrm{G}_{18}$ is written as the semi-direct product $\left(C_{3}^{I} \otimes C_{3}^{I}\right) \otimes C_{S}$, as reported by Ezra. ${ }^{30} \mathrm{~A}$ similar labeling scheme has been proposed by V. Van et al. for 2,5-dimethylthiophene, a molecule with two equivalent methyl rotors. ${ }^{31}$ The theoretical background was also described in detail by Altmann. ${ }^{32}$ The direct product $C_{3}^{I} \otimes C_{3}^{I}$ of the two intrinsic (superscript I) $C_{3}$ groups of the internal rotors, which is an invariant subgroup of $\mathrm{G}_{18}$, decomposes into five orbits $\left(\sigma_{1}, \sigma_{2}\right)$ under $C_{s}$. One representative of each orbit forms the first part of the symmetry label in column $\mathrm{S} 1$, e.g. (10)

The numbers $\sigma=0,1,2$ represent the three symmetry species $A, E_{a}, E_{b}$, respectively, of the group $\mathrm{C}_{3}$. They correspond to the transformation properties of the $\mathrm{C}_{3}$-adapted planar rotor wave functions $e^{i(3 k+\sigma) \varphi}$ with $k \in \mathbb{Z}$ and the torsional angle $\varphi$. Each orbit is associated with its little co-set. They form subgroups of $C_{s}$. Only for the orbit (00) the little co-set is $C_{s}$, while it is $C_{1}$ 
for all other orbits (01), (10), (11), and (12). The symmetry species of the little co-sets provide the second part of our symmetry labels in column S1.

Nuclear spin statistics. The total wave function $\psi_{\text {tot }}=\psi_{e l} \cdot \psi_{v i b} \cdot \psi_{\text {rotors }} \cdot \psi_{n s}$ must obey the Pauli principle, i.e. it changes sign under an odd number of fermion permutations (here protons) and retains sign under an even number of permutations. According to the character table, it belongs either to the $(00) \cdot \mathrm{A}^{\prime}$ or $(00) \cdot \mathrm{A}^{\prime \prime}$ species. There are 4096 nuclear spin functions arising from 12 protons in 34DMA with the representation $\Gamma_{\mathrm{ns}}=768(00) \cdot \mathrm{A}^{\prime} \oplus 256(00) \cdot \mathrm{A}^{\prime \prime} \oplus$ $512(10) \cdot \mathrm{A} \oplus 512(01) \cdot \mathrm{A} \oplus 256(11) \cdot \mathrm{A} \oplus 256(12) \cdot \mathrm{A}$. It is the product of the representations $\Gamma=16(00) \cdot A^{\prime}+8(10) \cdot A+8(01) \cdot A+4(11) \cdot A+4(12) \cdot A$ for the (1 23$)$ and (4 5 6) triples, $\Gamma=6(00) \cdot \mathrm{A}^{\prime}+2(00) \cdot \mathrm{A}^{\prime \prime}$ for the $(789)$ triple, and $\Gamma=8(00) \cdot \mathrm{A}^{\prime}$ for the three protons attached to the phenyl ring. The spin weights were determined by counting the number of allowed total wave functions for $\Gamma_{\mathrm{el}}=\Gamma_{\mathrm{vib}}=(00) \cdot \mathrm{A}^{\prime}$. The results are given in the last column of Table 1 (under $w t)$.

Selection rules. The dipole moment vector in space fixed coordinates is invariant under pure permutation operations but changes sign under inversion. Therefore, it transforms as $\Gamma_{\mu}=(00) \cdot \mathrm{A}^{\prime \prime}$. In the vibronic ground state, transitions between two rotorsional levels $i$ and $j$ are allowed if $\Gamma_{\mathrm{i}} \otimes \Gamma_{\mu} \otimes \Gamma_{\mathrm{j}} \supset(00) \cdot \mathrm{A}^{\prime}$. The resulting torsional components along with their spin statistical weights are given in Table 2 . They were determined by correlation with the molecular symmetry group $\mathrm{C}_{\mathrm{s}}(\mathrm{M})$ of the hypothetical rigid 34DMA. According to the spin statistical weight, the strength of the (11) and (12) torsional components is only half of other species.

The (00)· $\cdot \mathrm{A}^{\prime}$ species associates with the $K_{a} K_{c}=$ ee and oe transitions $(\mathrm{e}=$ even, o $=$ odd) and the $(00) \cdot \mathrm{A}^{\prime \prime}$ with the eo and oo transitions. Therefore, for the $(00)$ torsional components, two different $a$-type (ee $\leftrightarrow$ eo and oe $\leftrightarrow$ oo) and two different $b$-type transitions (ee $\leftrightarrow$ oo and eo $\leftrightarrow$ oe) are allowed with equal spin statistical weights. 
Obviously, if the quantum numbers $K_{a}$ and $K_{c}$ are known, the torsional states can be labeled conveniently by the first part $\left(\sigma_{1}, \sigma_{2}\right)$ of the full symmetry label. This abbreviated notation will be used throughout this paper.

Table 2. Spin statistical weights of torsional components of allowed transitions of 34DMA.

\begin{tabular}{|c|c|c|c|}
\hline \multicolumn{3}{|c|}{ Transitions } & $\mathrm{wt}$ \\
\hline$(00) \cdot A^{\prime}$ & $\leftrightarrow$ & $(00) \cdot A^{\prime \prime}$ & 1024 \\
\hline$(10) \cdot A$ & $\leftrightarrow$ & $(10) \cdot A$ & 1024 \\
\hline$(01) \cdot \mathrm{A}$ & $\leftrightarrow$ & $(01) \cdot A$ & 1024 \\
\hline$(11) \cdot \mathrm{A}$ & $\leftrightarrow$ & $(11) \cdot A$ & 512 \\
\hline$(12) \cdot A$ & $\leftrightarrow$ & $(12) \cdot A$ & 512 \\
\hline
\end{tabular}

\section{EXPERIMENT}

Experimental setup. The microwave spectra were recorded using a molecular jet Fourier transform microwave spectrometer operating in the frequency range from $2.0 \mathrm{GHz}$ to 26.5 GHz. ${ }^{33}$ The substance 34DMA with a stated purity of $99 \%$ was purchased from TCI Eschborn, Germany, and used without further purification. The colorless compound was trickled on a piece of pipe cleaner and inserted in a stainless steel tube mounted upstream the nozzle. Helium was used as carrier gas and the 34DMA-He mixture was expanded into the cavity at a backing pressure of 2 bar. A broadband scan from 8.5 to $13.5 \mathrm{GHz}$ was recorded by overlapping spectra with $0.25 \mathrm{MHz}$ step size. A portion of the scan is given in Figure 4. All signals observed in the broadband scan were remeasured at higher resolution where they appeared as doublets due to the Doppler effect. The line width average is $15 \mathrm{kHz}$, corresponding to a measurement accuracy of about $2 \mathrm{kHz}$. Figure 5 depicts a typical spectrum recorded at high resolution. 


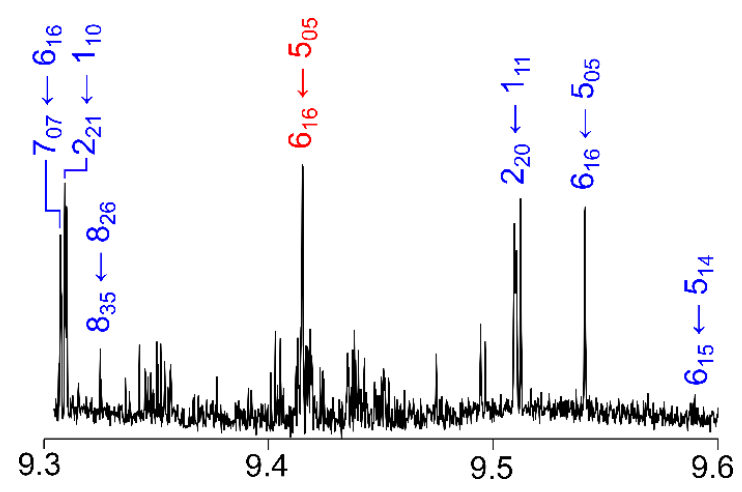

Figure 4. A portion of the broadband scan between 9.3 and $9.6 \mathrm{GHz}$. All signals are marked with their assigned quantum numbers (cis-34DMA in red, trans-34DMA in blue). The torsional splittings are not resolvable in the scan and therefore not marked.

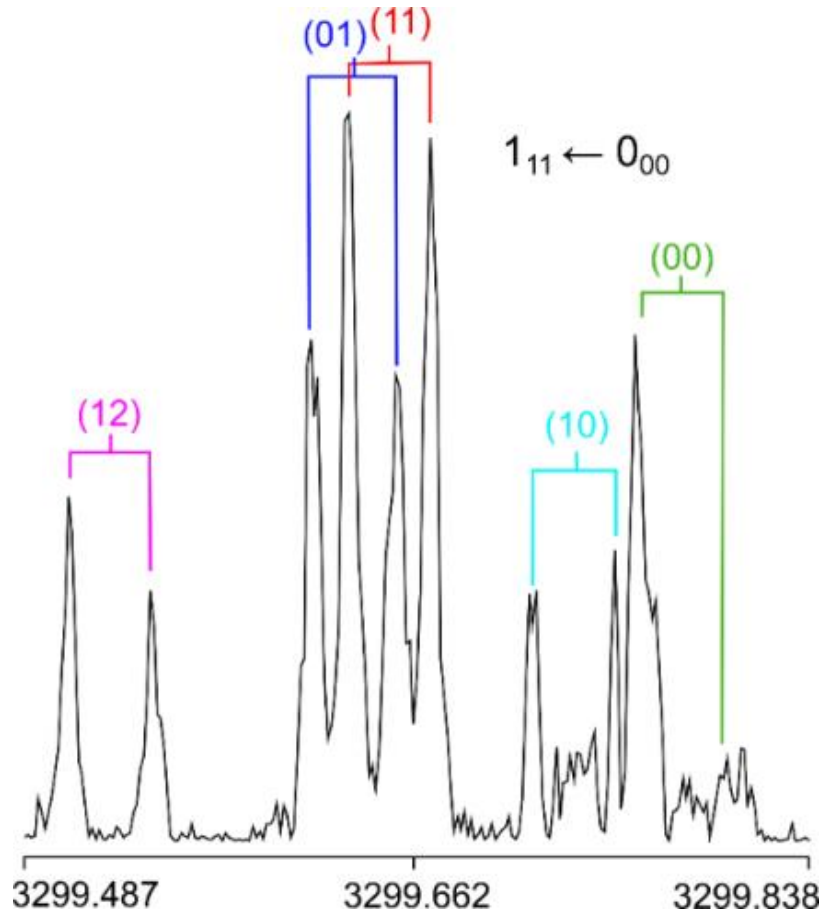

Figure 5. A typical spectrum of the $b$-type $1_{11} \leftarrow 0_{00}$ transition of cis-34DMA which splits into the (00), (10), (01), (11), and (12) torsional species. The frequency is in $\mathrm{MHz}$; the line width is approximately 15 $\mathrm{kHz}$ (full width at half-height), corresponding to a measurement accuracy of about $2 \mathrm{kHz}$. The splittings indicated with brackets are due to the Doppler effect. For this spectrum, 2653 free induction decays were co-added prior to Fourier transformation. 
Spectrum assignments. The assignment was started with the spectrum of the more stable trans conformer predicted to have the calculated dipole moment components $\mu_{a}=0.18 \mathrm{D}, \mu_{b}=-0.90$ $\mathrm{D}$, and $\mu_{c}=0.00 \mathrm{D}$. Initially, the internal rotations were neglected and trans-34DMA was treated as a rigid rotor, i.e. only the (00) species was considered. The theoretical spectrum was calculated using the program XIAM [34] in its rigid-rotor mode. Some transitions with $K_{a}=0$ and 1 could be assigned straightforwardly.

To analyze the methyl internal rotations, a two-top spectrum was predicted using the rotational constants from the rigid-rotor fit. The angles $\delta$ between the internal rotor axes and the principal $a$ axis and the barrier heights were taken from quantum chemical calculations. The assignment of the (01), (10), (11), and (12) torsional species was ambiguous because of the small torsional splittings. Therefore, first assignment attempts were performed for $b$-type transitions with $K_{a}=2$ and 3 featuring larger splittings. After the two-top fit was improved and provided better predictive power, lower $K_{a}$ transitions could also be assigned. Finally, $a$-type transitions were measured and fitted. Because of the small dipole moment component in the $a$ direction, only a limited number of $a$-type lines could be found. In total, $84(00), 84(01), 82$ (10), 83 (11), and 82 (12) torsional species lines were assigned and fitted to a standard deviation of $3.2 \mathrm{kHz}$ using the program XIAM. The molecular parameters of this fit are listed as Fit II in Table 3. A list of all fitted frequencies is available in Table S5 in the SI.

After the assignment of trans-34DMA, many lines remained in the broadband scan. They belong to the spectrum of cis-34DMA with the calculated dipole moment components $\mu_{a}=-0.15 \mathrm{D}, \mu_{b}=1.58 \mathrm{D}$, and $\mu_{c}=0.00 \mathrm{D}$. The assignments were carried out in the same manner as done for the trans conformer. The two-top XIAM fit with a standard deviation of $3.9 \mathrm{kHz}$ contains $61(00), 63(01), 65(10), 64(11)$, and 59 (12) torsional species lines. The molecular parameters of this fit are given as Fit $\mathbf{I}$ in Table 3, the frequency list in Table S6 in the SI. 
Table 3. Molecular parameters of the cis (I) and the trans (II) conformers of 34DMA as obtained with the program XIAM.

\begin{tabular}{|c|c|c|c|c|c|c|c|}
\hline Par. $^{a}$ & Unit & Fit I-(00) $)^{b}$ & Fit I & Calc. I $^{\mathrm{c}}$ & Fit II-(00) ${ }^{\mathrm{b}}$ & Fit II & Calc. II $^{\mathrm{c}}$ \\
\hline A & $\mathrm{MHz}$ & $2616.26903(81)$ & $2616.26800(29)$ & 2618.5487 & $2880.65424(58)$ & $2880.65412(24)$ & 2882.1896 \\
\hline B & $\mathrm{MHz}$ & $908.83530(46)$ & $908.83478(15)$ & 903.6490 & $855.06364(19)$ & $855.063955(74)$ & 850.7875 \\
\hline $\mathrm{C}$ & $\mathrm{MHz}$ & $683.42679(20)$ & $683.427404(67)$ & 680.3615 & $667.85553(12)$ & $667.855520(43)$ & 665.0552 \\
\hline$\Delta_{\mathrm{J}}$ & $\mathrm{kHz}$ & $0.0216(41)$ & $0.0180(14)$ & 0.01628 & $0.0116(12)$ & $0.01288(48)$ & 0.01131 \\
\hline$\Delta_{\mathrm{JK}}$ & $\mathrm{kHz}$ & $0.027(17)$ & $0.0101(57)$ & 0.00835 & $0.0413(56)$ & $0.0344(22)$ & 0.03646 \\
\hline$\Delta_{\mathrm{K}}$ & $\mathrm{kHz}$ & $0.288(46)$ & $0.268(15)$ & 0.24894 & $0.401(48)$ & $0.392(19)$ & 0.34957 \\
\hline$\delta_{\mathrm{J}}$ & $\mathrm{kHz}$ & $0.0065(23)$ & $0.00500(76)$ & 0.00464 & $0.00298(69)$ & $0.00339(27)$ & 0.00269 \\
\hline$\delta_{\mathrm{K}}$ & $\mathrm{kHz}$ & $0.208(43)$ & $0.071(15)$ & 0.04495 & $0.025(20)$ & $0.0394(76)$ & 0.04155 \\
\hline $\mathrm{V}_{3,1}$ & $\mathrm{~cm}^{-1}$ & $430.00^{\mathrm{d}}$ & $430.00(37)$ & 364.26 & $499.64^{\mathrm{f}}$ & 499.64(26) & 421.38 \\
\hline $\mathrm{V}_{3,2}$ & $\mathrm{~cm}^{-1}$ & $467.90^{\mathrm{d}}$ & $467.90(17)$ & 393.22 & $533.54^{\mathrm{f}}$ & $533.54(22)$ & 444.70 \\
\hline $\mathrm{F}_{0,1}$ & $\mathrm{GHz}$ & $162.23^{\mathrm{d}}$ & $162.23^{\mathrm{e}}$ & 162.23 & $162.40^{\mathrm{f}}$ & $162.40^{\mathrm{e}}$ & 162.40 \\
\hline $\mathrm{F}_{0,2}$ & $\mathrm{GHz}$ & $163.77^{\mathrm{d}}$ & $163.77^{\mathrm{e}}$ & 163.77 & $163.70^{\mathrm{f}}$ & $163.70^{\mathrm{e}}$ & 163.70 \\
\hline$\angle\left(\mathrm{i}_{1}, \mathrm{a}\right)$ & $\circ$ & $117.14^{\mathrm{d}}$ & $117.14(13)$ & 120.38 & $131.74^{\mathrm{f}}$ & $131.74(14)$ & 135.58 \\
\hline$\angle\left(\mathrm{i}_{2}, \mathrm{a}\right)$ & $\circ$ & $176.3^{\mathrm{d}}$ & $176.3(15)$ & 177.50 & $158.85^{\mathrm{f}}$ & $158.85(30)$ & 162.50 \\
\hline$\angle\left(\mathrm{i}_{1}, \mathrm{~b}\right)$ & $\circ$ & $27.14^{\mathrm{d}}$ & $27.14(13)$ & 30.38 & $41.74^{\mathrm{f}}$ & $41.74(14)$ & 45.58 \\
\hline$\angle\left(\mathrm{i}_{2}, \mathrm{~b}\right)$ & $\circ$ & $86.3^{\mathrm{d}}$ & $86.3(15)$ & 92.50 & $111.15(30)^{\mathrm{f}}$ & $111.15(30)$ & 107.50 \\
\hline$\angle\left(\mathrm{i}_{1}, \mathrm{c}\right)$ & $\circ$ & $90.00^{\mathrm{d}}$ & $90.00^{\mathrm{g}}$ & 90.00 & $90.00^{\mathrm{f}}$ & $90.00^{\mathrm{g}}$ & 89.99 \\
\hline$\angle\left(\mathrm{i}_{2}, \mathrm{c}\right)$ & $\circ$ & $90.00^{\mathrm{d}}$ & $90.00^{\mathrm{g}}$ & 89.98 & $90.00^{f}$ & $90.00^{\mathrm{g}}$ & 90.00 \\
\hline$N^{\mathrm{h}}$ & & 61 & 312 & & 84 & 415 & \\
\hline$\sigma^{\mathrm{i}}$ & $\mathrm{kHz}$ & 5.0 & 3.9 & & 3.7 & 3.2 & \\
\hline
\end{tabular}

a All parameters are given with one standard uncertainty in parentheses. Watson's A reduction and $I^{r}$ representation were used. ${ }^{b}$ Fit including only the (00) torsional species. ${ }^{c}$ Calculated at the B3LYP/6$311++\mathrm{G}(\mathrm{d}, \mathrm{p})$ level of theory. Geometry parameters obtained from geometry optimizations, centrifugal distortion constants from anharmonic frequency calculations. ${ }^{\mathrm{d}}$ Fixed to the fitted value of Fit I. ${ }^{\mathrm{e}}$ Fixed to the calculated value. ${ }^{\mathrm{f}}$ Fixed to the fitted value of Fit II. ${ }^{\mathrm{g}}$ Fixed due to $\mathrm{C}_{\mathrm{s}}$ symmetry. ${ }^{\mathrm{h}}$ Number of lines. ${ }^{\mathrm{i}}$ Standard deviation of the fit.

\section{RESULTS \& DISCUSSION}

Using the program XIAM, the spectra of cis-34DMA and trans-34DMA including 312 and 415 lines, respectively, were fitted to standard deviations close to measurement accuracy, yielding accurately determined molecular parameters. All calculated rotational constants are in very 
satisfactory agreement with the experimental values, whereby the deviations are less than 5 $\mathrm{MHz}$ for both conformers. The centrifugal distortion constants obtained from anharmonic frequency calculations at the B3LYP/6-311++G(d,p) level of theory are also in good agreement with the experimental values. Since the phenyl ring of 34DMA is quite rigid, all centrifugal distortion constants are small.

It should be mentioned that all predicted rotational constants refer to the equilibrium $r_{e}$ structure, while the experimental constants represent the vibrational ground state $r_{0}$ structure. Theoretical rotational constants of the $r_{0}$ structure can be obtained by anharmonic frequency calculations. However, they show larger deviations to the experimental values compared to those of the $r_{e}$ structure, because the calculations in electronic structure packages are generally not able to describe the LAMs correctly. The potential is assumed to be a quartic potential in Cartesian coordinates rather than a triple-well potential in internal coordinates. For cis- and trans-34DMA the centrifugal distortion corrected rotational constants of the ground vibrational state are $A_{0}=2585.740 \mathrm{MHz}, B_{0}=900.041 \mathrm{MHz}$, and $C_{0}=676.459 \mathrm{MHz}$, and $A_{0}=2846.265$ $\mathrm{MHz}, B_{0}=846.597 \mathrm{MHz}$, and $C_{0}=660.936 \mathrm{MHz}$, respectively.

Although the standard deviations obtained from Fit I and Fit II are good, they are still by a factor of two larger than the estimated measurement accuracy of about $2 \mathrm{kHz}$. Therefore, an additional fit for each conformer was performed, where only the (00) species lines were taken into account, while all internal rotation parameters were fixed to those of the global fits. These fits are given as Fit (00) in Table 3. The standard deviations of Fit (00) are not significantly lower than those of Fit I and Fit II, showing that the deviations of about $4 \mathrm{kHz}$ of the global fits are not due to the lack of parameters in the Hamiltonian. Some combination difference loops were calculated for both conformers, and they also indicate differences of about $4 \mathrm{kHz}$. 
Because of the strong correlation with $V_{3}, F_{0}$ was fixed to the calculated values in both Fit I and Fit II. The barrier heights of the meta and para methyl tops are 430.00(37) and 467.90(17) $\mathrm{cm}^{-1}$, respectively, for cis-34DMA, as well as 499.64(26) and 533.54(22) $\mathrm{cm}^{-1}$, respectively, for trans-34DMA, leading to the following conclusions: (i) The torsional barriers of both methyl groups are higher in the trans than in the cis conformer, and (ii) The barrier of the meta methyl top is lower than that of the para methyl top in both conformers. The first observation hints that conformation effects, corresponding to different orientations of the methoxy group, on the ring methyl internal rotations are present. On the other hand, the trend is in contrast to the trend reported in 3,4-DMBA, stating that the barriers to internal rotation are higher in the anti (trans) than in the syn (cis) conformer for both methyl tops (see Figure 6). ${ }^{18}$ As discussed in ref. 37, there are two factors that affect the height of a methyl barrier: the steric hindrance and the electronic effect. In the two conformers of both, 34DMA and 3,4-DMBA, the steric effect does not play a significant role because of the long distance between the two substituents and the methoxy group. Therefore electronic properties are more likely to be responsible for the inversion in the barrier heights in the trans and cis configurations, because for 3,4-DMBA the $\pi$-system of the ring is extended by the carbonyl group. On the other hand, the barriers to internal rotation of the meta and para methyl groups of 34DMA are by an order of magnitude higher than the respective values found in $m$ - and $p$-methylanisole. A similar trend was found in 3,4-DMBA, as shown in Figure 6. This is clearly due to steric hindrance arising from the close proximity of the two ring methyl groups. 
<smiles>COc1ccc(C)c(C)c1</smiles>

(1) cis

m: $430 \mathrm{~cm}^{-1}$

p: $468 \mathrm{~cm}^{-1}$<smiles>Cc1ccc(C=O)cc1C</smiles>

(4) cis<smiles>COc1ccc(C)c(C)c1</smiles>

(1) trans<smiles>COc1cccc(C)c1</smiles>

(2) cis<smiles>COc1cccc(C)c1</smiles>

(2) trans<smiles>COc1ccc(C)cc1</smiles>

(3)

\section{m: $500 \mathrm{~cm}^{-1}$}

$56 \mathrm{~cm}^{-1}$

$36 \mathrm{~cm}^{-1}$

$49 \mathrm{~cm}^{-1}$

p. $534 \mathrm{~cm}^{-1}$<smiles>Cc1ccc(C=O)cc1C</smiles><smiles>Cc1cccc(C=O)c1</smiles>

(5) cis<smiles>Cc1cccc(C=O)c1</smiles>

(5) trans<smiles>Cc1ccc(C=O)cc1</smiles>

(6)

$$
\begin{array}{lllll}
\text { m: } 508 \mathrm{~cm}^{-1} & \text { m: } 454 \mathrm{~cm}^{-1} & \text { m: } 36 \mathrm{~cm}^{-1} & \text { m: } 5 \mathrm{~cm}^{-1} & \text { p: } 28 \mathrm{~cm}^{-1} \\
\text { p: } 551 \mathrm{~cm}^{-1} & \text { p: } 481 \mathrm{~cm}^{-1} & & &
\end{array}
$$

Figure 6. Torsional barriers in $\mathrm{cm}^{-1}$ of the meta and para methyl groups in methylanisoles and methylbenzaldehydes. (1) 3,4-dimethylanisole (this work), (2) $m$-methylanisole, ${ }^{23}$ (3) $p$-methylanisole, ${ }^{24}$ (4) 3,4-dimethylbenzaldehyde, ${ }^{18}$ (5) $m$-methylbenzaldehyde, ${ }^{35}$ (6) $p$-methylbenzaldehyde. ${ }^{36}$

The calculated torsional barrier of the meta methyl group is $364.26 \mathrm{~cm}^{-1}$ for cis-34DMA and $421.38 \mathrm{~cm}^{-1}$ for trans-34DMA, which are in the same order of magnitude of the experimental values, but lower in both cases (see Table 3 for comparison). For the para methyl group, the values of $393.22 \mathrm{~cm}^{-1}$ for cis-34DMA and $444.70 \mathrm{~cm}^{-1}$ were determined for trans34DMA. The experimental values of $467.90(17) \mathrm{cm}^{-1}$ and $533.54(22) \mathrm{cm}^{-1}$, respectively, are again higher than the theoretical ones.

Finally, it should be mentioned that after the spectra of the trans and the cis conformer of 34DMA have been assigned, no unidentified lines remain in the broadband scan. This indicates that complexes, isotopologues in natural abundances, and torsional excited states are not present under our measurement conditions. 


\section{CONCLUSIONS}

The spectra of two conformers of 3,4-dimethylanisole measured under molecular jet conditions were assigned using a combination of Fourier transform microwave spectroscopy, group theory, and quantum chemistry. The splittings of each rotational transition into five torsional species arising from the internal rotations of two non-equivalent ring methyl groups were observed. A labelling scheme for the group $G_{18}$ written as the semi-direct product $\left(C_{3}^{I} \otimes C_{3}^{I}\right) \otimes C_{s}$ was introduced. Quantum chemical calculations at the B3LYP/6-311++G(d,p) level of theory yielded reasonable structural parameters, as well as barrier heights in the expected order of magnitude. The barriers to internal rotation are mainly influenced by electronical effects and steric effects arising from the adjacent positions of the ring methyl groups. Steric effect arising from different orientations of the methoxy group is not significant because of the long distance to the rotors, but also observable, showing that conformation effects on the ring methyl internal rotations are present.

\section{ASSOCIATED CONTENT}

Supporting Information. Fourier coefficients of the potential energy curves, Cartesian coordinates of the trans and cis conformers, figure and Fourier coefficients of the 2D-PES, lists of fitted frequencies along with their residuals.

\section{ACKNOWLEDGMENTS}

Simulations were performed with computing resources granted by RWTH Aachen University under project <thes0248>. W.S. thanks the Université Paris-Est for an invited researcher grant which enabled him to work at the Université Paris-Est Créteil. 


\section{REFERENCES}

(1) Andresen, M.; Kleiner, I.; Schwell, M.; Stahl, W.; Nguyen, H. V. L. Acetyl Methyl Torsion in Pentan-2-one As Observed by Microwave Spectroscopy. J. Chem. Phys. A. 2018, 122, 7071-7078.

(2) Eibl, K.; Stahl, W.; Kleiner, I.; Nguyen, H. V. L. Conformational Effect on the Almost Free Internal Rotation in 4-Hexyn-3-ol Studied by Microwave Spectroscopy and Quantum Chemistry. J. Chem. Phys. 2018, 149, 144306.

(3) Melandri, S.; Maris, A.; Favero, L. B. The Double Donor/Acceptor Role of the $\mathrm{NH}_{3}$ Group: Microwave Spectroscopy of the Aminoethanol-Ammonia Molecular Complex. J. Mol. Phys. 2010, 108, 2219-2223.

(4) Shubert, V. A.; Schmitz, D.; Schnell, M.; Communication through the Phenyl Ring: Internal Rotation and Nuclear Quadrupole Splitting in p-Halotoluenes. J. Mol. Phys. 2013, 111, 2189-2197.

(5) Nguyen, H. V. L.; Stahl, W. The Effects of Nitrogen Inversion Tunneling, Methyl Internal Rotation, and ${ }^{14} \mathrm{~N}$ Quadrupole Coupling Observed in the Rotational Spectrum of Diethyl Amine, J. Chem. Phys. 2011, 135, 024310.

(6) Ferres, L.; Mouhib, H.; Stahl, W.; Schwell, M.; Nguyen, H. V. L. Molecular Structure and Ring Tunneling of Phenyl Formate as Observed by Microwave Spectroscopy and Quantum Chemistry, J. Mol. Spectrosc. 2017, 337, 59-64.

(7) Cabezas, C.; Varela, M.; Caminati, W.; Mata, S.; López, J. C.; Alonso, J. L. The Two Conformers of Acetanilide Unraveled Using LA-MB-FTMW Spectroscopy, J. Mol. Spectrosc. 2011, 268, 42-46.

(8) Ilyushin, V. V.; Johnson, A. M.; Hohl, J.; Cloessner, E. A.; Lovas, F. J. Lavrich, R. J. Isotopic Dependence of the Hydrogen-Transfer-Triggered Methyl-Group Rotation in Deuterated 5-Methyltropolone. J. Mol. Spectrosc. 2018, 343, 76-80. 
(9) Nair, K. P. R.; Herbers, S.; Obenchain, D. A.; Grabow, J.-U.; Lesarri, A. The Low Internal Rotation Barriers of Halogenated Toluenes: Rotational Spectrum of 2,4Difluorotoluene. J. Mol. Spectrosc. 2018, 344, 21-26.

(10) Lees, R. M.; Xu, L.-H.; Guislain, B. G.; Reid, E. M.; Twagirayezu, S.; Perry, D. S.; Dawadi, M. B.; Thapaliya, B. P. Billinghurst, B. E. Torsion-Rotation Structure and Quasi-Symmetric-Rotor Behavior for the $\mathrm{CH}_{3} \mathrm{SH}$ Asymmetric $\mathrm{CH}_{3}$-Bending and $\mathrm{C}-\mathrm{H}$ Stretching Bands of E Parentage. J. Mol. Spectrosc. 2018, 343, 18-27.

(11) Hakiri, R.; Derbel, N.; Nguyen, H. V. L.; Mouhib, H. Communication through the Furan Ring: Conformational Effect on the Internal Rotation of 5-Methyl Furfural Studied by Microwave Spectroscopy. Phys. Chem. Chem. Phys. 2018, 20, 25577-25582.

(12) Ohashi, N.; Tsunekawa, S.; Takagi, K.; Hougen, J. T. Microwave Spectrum of Methyl Amine: Assignment and Analysis of the Torsional State. J. Mol. Spectrosc. 1989, $137,33-46$.

(13) Van, V.; Stahl, W.; Nguyen, H. V. L. The Structure and Torsional Dynamics of Two Methyl Groups in 2-Acetyl-5-methylfuran as Observed by Microwave Spectroscopy. ChemPhysChem 2016, 17, 3223-3228.

(14) Nguyen, H. V. L.; Kleiner, I.; Shipman, S. T.; Mae, Y.; Hirose, K.; Hatanaka, S.; Kobayashi, K. Extension of the Measurement, Assignment, and Fit of the Rotational Spectrum of the Two-Top Molecule Methyl Acetate. J. Mol. Spectrosc. 2014, 299, $17-21$

(15) Van, V; Bruckhuisen, J.; Stahl, W.; Ilyushin, V.; Nguyen, H. V. L. The Torsional Barriers of Two Equivalent Methyl Internal Rotations in 2,5-Dimethylfuran Investigated by Microwave Spectroscopy. J. Mol. Spectrosc. 2018, 343, 121-125.

(16) Plusquellic, D. F.; Kleiner, I.; Demaison, J.; Suenram, R. D.; Lavrich, R. J.; Lovas, F. J.; Fraser, G. T.; Ilyushin, V. V. The Microwave Spectrum of a Two-Top Peptide 
Mimetic: The $N$-acetyl Alanine Methyl Ester Molecule. J. Chem. Phys. 2006, 125, 104312.

(17) Nguyen, H. V. L.; Stahl, W.; Kleiner, I. Structure and Rotational Dynamics of Methyl Propionate Studied by Microwave Spectroscopy. Mol. Phys. 2012, 110, 2035-2042.

(18) Tudorie, M.; Kleiner, I.; Jahn, M.; Grabow, J.-U.; Goubet, M.; Pirali, O. Coupled Large Amplitude Motions: A Case Study of the Dimethylbenzaldehyde Isomers. J. Phys. Chem. A. 2013, 117, 13636-13647.

(19) Rudolph, H. D.; Walzer, K.; Krutzik, I. Microwave Spectrum, Barrier for Methyl Rotation, Methyl Conformation, and Dipole Moment of ortho-Xylene. J. Mol. Spectrosc. 1973, 47, 314-339.

(20) Thomsen, C.; Dreizler, H. The Microwave Spectra of $m$-Xylene and $m$-Xylene-d $\mathrm{d}_{10}$. Determination of the Low Methyl Internal Rotation Barrier. Z. Naturforsch. 2001, $56 a, 635-640$.

(21) Ferres, L.; Truong, K.-N.; Stahl, W.; Nguyen, H. V. L. Interplay between Microwave Spectroscopy and X-ray Diffraction: The Molecular Structure and Large Amplitude Motions of 2,3-Dimethylansiole. ChemPhysChem 2018, 19, 1781-1788.

(22) Ferres, L.; Mouhib, H.; Stahl, W.; Nguyen, H. V. L. Methyl Internal Rotation in the Microwave Spectrum of $o$-Methyl Anisole. ChemPhysChem. 2017, 18, 1855-1859.

(23) Ferres, L.; Stahl, W.; Nguyen, H. V. L. Conformational Effects on the Torsional Barriers in $m$-Methylanisole Studied by Microwave Spectroscopy. J. Chem. Phys. 2018, 148, 124304.

(24) Ferres, L.; Stahl, W.; Kleiner, I.; Nguyen, H. V. L. The Effect of Internal Rotation in pMethyl Anisole Studied by Microwave Spectroscopy. J. Mol. Spectrosc. 2018, 343, 4449. 
(25) Frisch, M. J.; Trucks, G. W.; Schlegel, H. B.; Scuseria, G. E.; Robb, M. A.; Cheeseman, J. R.; Scalmani, G.; Barone, V.; Mennucci, B.; Petersson G. A. et al. Gaussian 09, Gaussian, Inc., Wallingford CT, 2009.

(26) Schlegel, H. B. Optimization of Equilibrium Geometries and Transition Structures. J. Comput. Chem. 1982, 3, 214-218.

(27) Reinhold, B., Finneran, I. A.; Shipman, S. T. Room Temperature Chirped-pulse Fourier Transform Microwave Spectroscopy of Anisole. J. Mol. Spectrosc. 2011, 270, 89-97.

(28) Dreizler, H. Gruppentheoretische Betrachtungen zu den Mikrowellenspektren von Molekülen mit zwei behindert drehbaren dreizähligen-symmetrischen Molekülgruppen. Z. Naturforsch. 1961, 16a, 1354-1367.

(29) Bunker, P. R.; Jensen, P. Molecular Symmetry and Spectroscopy, $2^{\text {nd }}$ edition, $N R C$ Research Press, Ottawa, Ontario, Canada 2006.

(30) Ezra, G. S. Symmetry Properties of Molecules, Lecture Notes in Chemistry, Springer-Verlag, Berlin, Heidelberg, New York 1982, 28.

(31) Van, V.; Stahl, W.; Nguyen, H. V. L. Two Equivalent Methyl Internal Rotations in 2,5-Dimethylthiophene Investigated by Microwave Spectroscopy, Phys. Chem. Chem. Phys. 2015, 48, 32111-32114.

(32) Altmann, S. L. Induced Representations in Crystals and Molecules, Point, Space and Nonrigid Molecule Groups, Academic Press, London, New York, San Francisco 1977.

(33) Grabow, J.-U.; Stahl, W.; Dreizler, H. A Multioctave Coaxially Orientated BeamResonator Arrangement Fourier-Transform Microwave Spectrometer. Rev. Sci. Instrum. 1996, 67, 4072-4084.

(34) Hartwig, H.; Dreizler, H. The Microwave Spectrum of trans-2,3-Dimethyloxirane in Torsional Excited States. Z. Naturforsch. 1996, 51a, 923-932. 
(35) Shirar, A. J.; Wilcox, D. S.; Hotopp, K. M.; Storck, G. L.; Kleiner, I.; Dian, B. C. Impact of Molecular Conformation on Barriers to Internal Methyl Rotation: The Rotational Spectrum of m-Methylbenzaldehyde. J. Phys. Chem. A 2010, 114, 12187-12194.

(36) Saal, H.; Grabow, J.-U.; Hight Walker, A. R.; Hougen, J. T.; Kleiner, I.; Caminati, W. Microwave Study of Internal Rotation in para-Tolualdehyde: Local Versus Global Symmetry Effects at the Methyl-rotor Site. J. Mol. Spectrosc. 2018, 351, $55-61$.

(37) Jabri, A.; Van, V.; Nguyen, H. V. L.; Stahl, W.; Kleiner, I. Probing the Methyl Torsional Barriers of the $E$ and $Z$ Isomers of Butadienyl Acetate by Microwave Spectroscopy. ChemPhysChem 2016, 17, 2660-2665.

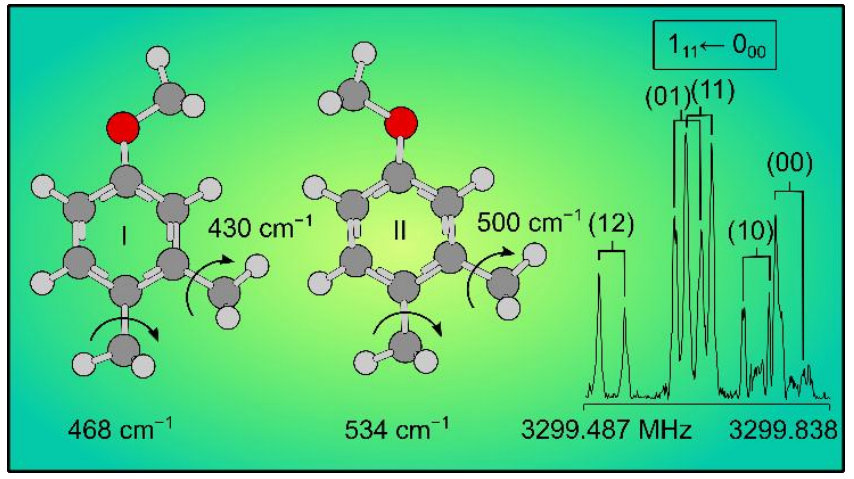

TOC Graphic 